\title{
Acupuncture in ambulatory anesthesia: a review
}

This article was published in the following Dove Press journal:

Ambulatory Anesthesia

7 September 2015

Number of times this article has been viewed

\author{
Arne Johan Norheim' \\ Ingrid Liodden' \\ Terje Alræk ${ }^{1,2}$ \\ 'National Research Center in \\ Complementary and Alternative \\ Medicine (NAFKAM), Department \\ of Community Medicine, Faculty \\ of Health Sciences, University of \\ Tromsø - The Arctic University of \\ Norway, Troms $\varnothing,{ }^{2}$ The Norwegian \\ School of Health Sciences, Institute \\ of Acupuncture, Kristiania University \\ College, Oslo, Norway
}

Background: Post-anesthetic morbidities remain challenging in our daily practice of anesthesia. Meta-analyses and reviews of acupuncture and related techniques for postoperative nausea and vomiting (POVN) and postoperative vomiting (POV) show promising results while many clinicians remain skeptical of the value of acupuncture. Given the interest in finding safe nonpharmacological approaches toward postoperative care, this body of knowledge needs to be considered. This review critically appraises and summarizes the research on acupuncture and acupressure in ambulatory anesthesia during the last 15 years.

Methods: Articles were identified through searches of Medline, PubMed, and Embase using the search terms "acupuncture" or "acupuncture therapy" in combination with "ambulatory anesthesia" or "ambulatory surgery" or "day surgery" or "postoperative". A corresponding search was done using "acupressure" and "wristbands". The searches generated a total of 104, 118, and 122 references, respectively.

Results: Sixteen studies were included; eight studies reported on acupuncture and eight on acupressure. Nine studies found acupuncture or acupressure effective on primary endpoints including postoperative nausea and vomiting, postoperative pain, sore throat, and emergence agitation. Four studies found acupuncture had a similar effect to antiemetic medication.

Conclusion: Overall, the studies were of fairly good quality. A large proportion of the reviewed papers highlights an effect of acupuncture or acupressure on postoperative morbidities in an ambulatory setting. However, one should bear in mind that research on acupuncture/acupressure in an ambulatory setting contributes to ambiguous conclusions. Hence, we have addressed some of the issues related to this diversity in acupuncture research.

Keywords: acupressure, anesthesia, postoperative, nausea, pain, complementary and alternative medicine

\section{Introduction}

A growing awareness of quality in health care has called for a focus on post-anesthetic morbidities, which still remain challenging in our daily practice of anesthesia, such as nausea, vomiting, and pain. ${ }^{1-3}$ The incidence of postoperative nausea and vomiting (PONV) is shown to be in the range of $17 \%$ to $34 \%,{ }^{4,5}$ with numbers as high as $82 \%$ reported in at-risk patients. ${ }^{6}$ Postoperative pain (POP) has been experienced by $30 \%$ of patients, and as many as 50\% experienced mild POP. ${ }^{7}$ A gradually pain-free state was reported by $36 \%$ of patients. $^{8}$

The development of new surgical techniques and new anesthetic drugs has made ambulatory surgery a rapidly changing health care activity. Despite continued efforts in both treatment and prevention of PONV and POP, these symptoms continue after
Correspondence: Arne Johan Norheim National Research Center in

Complementary and Alternative Medicine (NAFKAM), Department of Community Medicine, Faculty of Health Sciences, University of Troms $\varnothing$ - The Arctic University of Norway, N-9038 Tromsø, Norway

Phone +47 77646650

Email arne@avital.no 
both inpatient and ambulatory surgical procedures. Since ambulatory patients are discharged to home a few hours postoperatively, it is particularly important to ensure optimal and adequate emetic and pain management.

The numerous receptors involved support the suggestion of a multifactorial etiology of emesis ${ }^{9}$ and may explain why antiemetics are only partially effective in preventing PONV. In addition, antiemetic drugs can cause unpleasant adverse events, such as agitation and restlessness, sedation and delayed awakening, extrapyramidal reactions, headache, blurred vision, dry mouth, and tachycardia. ${ }^{10}$ Opioids intraoperatively increase the incidence of PONV and prolong recovery, ${ }^{11}$ and nonsteroidal anti-inflammatory drugs can cause ulcers in the gastrointestinal tract. ${ }^{12}$ Since the adverse events are dose dependent, Kehlet and Dahl suggested in their seminal paper ${ }^{13}$ that a combination of medications acting through different mechanisms allows lower doses of analgesics, resulting in better pain control and fewer adverse events. Multimodal analgesic treatment is thus recommended for POP. ${ }^{14,15}$

The awareness of and interest in complementary and alternative medicine is increasing among patients and health care providers worldwide. ${ }^{16-18}$ Acupuncture and acupressure are therapeutic modalities often used for the treatment of nausea and vomiting due to morning sickness, ${ }^{19}$ chemotherapy, ${ }^{20}$ and PONV. ${ }^{21}$ Several meta-analyses and reviews of acupuncture and related techniques for PONV and POV have been published. ${ }^{21-30}$ Still, many clinicians remain skeptical of the value of acupuncture. They argue that the evidence is far from convincing, lacks rigor, and is conflicting. ${ }^{31-34}$

Bodily acupuncture is in general associated with minor adverse events, ${ }^{35,36}$ although serious adverse events such as pneumothorax and needle fracture have been reported. ${ }^{35}$ However, in general, acupuncture in the hands of qualified practitioners, and in particular acupressure, is regarded as a safe therapy.

Given the interest in finding safe non-pharmacological approaches toward postoperative care, this body of knowledge needs to be considered. This review critically appraises and summarizes the research on acupuncture and acupressure in ambulatory anesthesia during the last 15 years.

\section{Materials and methods}

\section{Search strategy}

Articles were identified through computerized literature searches. Medline, PubMed, and Embase were searched for publications from January 1, 2000 to February 1, 2015, using the search terms "acupuncture" or "acupuncture therapy" in combination with "ambulatory anesthesia" or "ambulatory surgery" or "day surgery" or "postoperative". All fields, keywords, and Medical Subject Headings (MeSH) terms were included. Filters were English language, human, and clinical trial. A corresponding search was performed using the terms "acupressure" and "wristbands".

\section{Selection criteria}

The selection criteria included randomized controlled trials (RCTs) are published in peer-reviewed journals on needling acupuncture or acupressure for the alleviation of postoperative morbidities. Further criteria were studies in general or regional anesthesia in ambulatory settings. We decided to include articles published after the year 2000, due to the fact that anesthesiology and surgical techniques, and methodology in acupuncture research, have been developed further in recent decades.

\section{Selection of papers}

The search performed in Medline, PubMed, and Embase provided a total of 104, 118, and 122 references, respectively. We excluded articles that were not relevant to the aim of this review, pilot studies, most inpatient studies, studies using acupuncture injection, transcutaneous electro-acustimulation, electroacupuncture, ear and hand acupuncture, non-surgery studies, non-clinical studies, and studies of surgery performed under local anesthesia.

The literature search identified 15 peer-reviewed RCTs that met the inclusion criteria. Further, one more study seemed to be relevant. To clarify an inclusion criterion, we emailed the corresponding author of that study, but received no response. Hence, the study was excluded. In addition to the 15 published studies, we included one relevant article in press, ${ }^{37}$ meaning the review encompassed, in total, 16 RCTs. Figure 1 displays a flow diagram of the paper-selection process.

The literature search also identified numerous other studies on acupuncture-related techniques other than needling acupuncture and acupressure. These involved therapeutic modalities including acupuncture injection, ${ }^{38,39}$ electroacupuncture, ${ }^{40,41}$ transcutaneous electro acupoint stimulation, ${ }^{42,43}$ auricular acupuncture, ${ }^{44,45}$ Korean hand acupressure, ${ }^{46,47}$ and capsicum plaster used on acupuncture points. ${ }^{48,49}$ These studies were excluded as they did not meet the selection criteria.

The conceptual framework in traditional Chinese medicine (TCM) explains the effect of acupuncture and acupressure based on the belief that an individual's well-being depends on the balance of energy in the body. According to TCM, energy free flows within the body and also along paths 

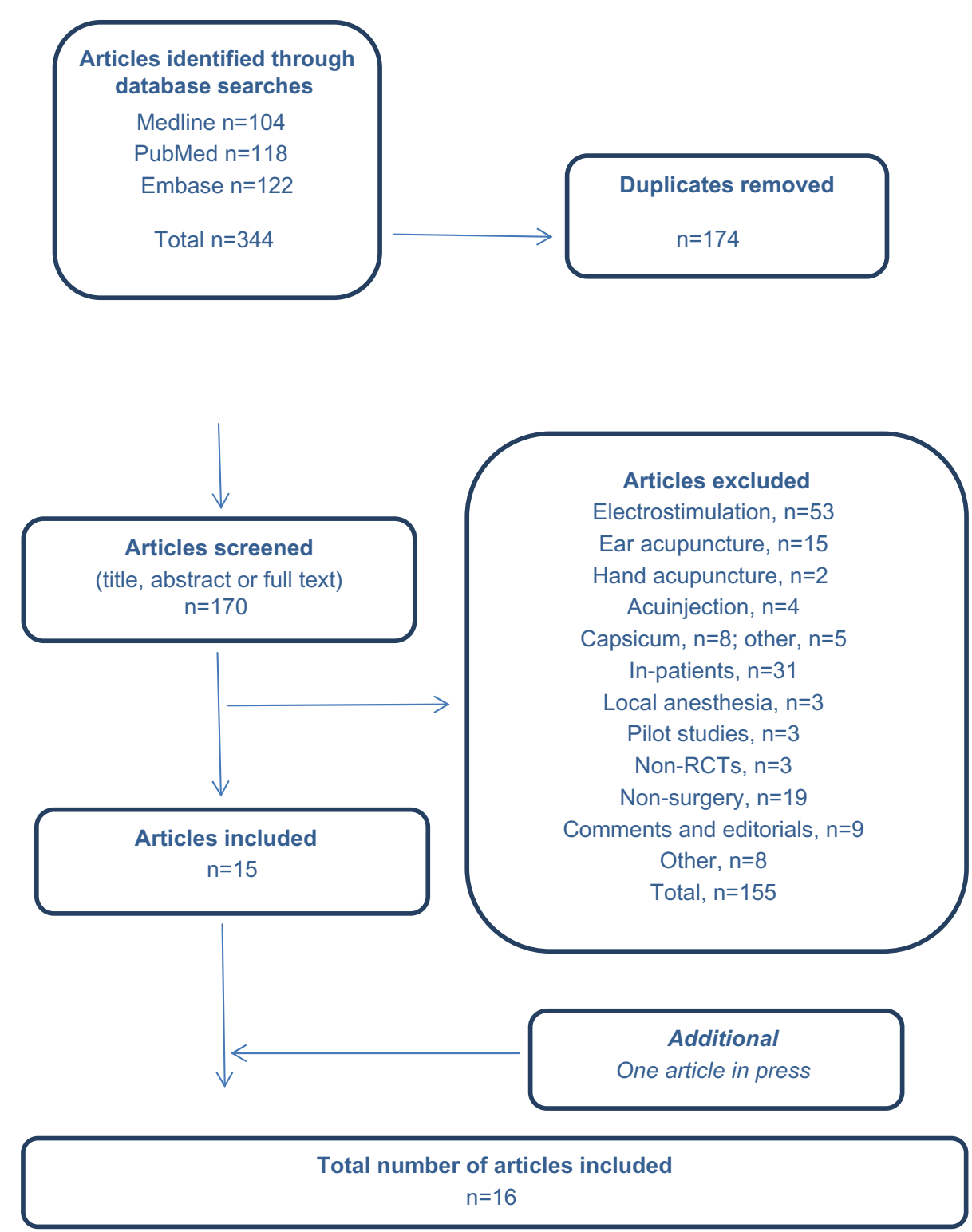

Figure I Flow diagram for article identification and selection.

Abbreviation: RCTs, randomized controlled trials.

referred to as meridians. Acupuncture techniques restore the balance of energy through the application of acupoints, hence manipulating the flow of energy in the meridians or in the body. ${ }^{50}$ The acupuncture point Pericardium 6 (PC6 Neiguan) is a Chinese meridian point frequently used for the treatment of nausea and vomiting. The present review refers to acupuncture points according to World Health Organization-proposed Standard International Acupuncture Nomenclature. $^{51}$

\section{Results}

We included in total 16 studies in our review, eight reporting on acupuncture and eight on acupressure. All studies reported on surgery performed under general anesthesia. Five studies included children, among them three were on acupuncture, one study was on combined acupuncture/acupressure, and one was on acupressure. While acupressure more or less exclusively is used for PONV in ambulatory anesthesia, acupuncture is in addition used for other morbidities, such as pain, emergence agitation, sore throat, and hypothermia (Tables 1 and 2).

Nine studies out of 16 found acupuncture or acupressure effective on primary endpoints including PONV, POP, sore throat, and emergence agitation. ${ }^{52-60}$ One study found acupuncture partly effective for pain ${ }^{61}$ and one study found acupressure effective for vomiting, but not nausea. ${ }^{62}$ 
Four studies found acupuncture having a similar effect as antiemetics..$^{52,54,55,60}$ Two out of eight studies on acupuncture and three out of eight studies on acupressure did not find any effect on the selected primary endpoints. . $^{37,63-66}$

Eleven studies reported on PONV as a primary endpoint using acupuncture/acupressure at point PC6, and among these eleven studies one study ${ }^{60}$ combined PC6 with CV13. ${ }^{37,52-55,60,62-66}$ Three studies ${ }^{57,58,61}$ reported on POP as primary endpoint. One of these three studies used acupuncture points GV2, GV20, BL30, BL57, PC6, and Nei Ting. ${ }^{61}$ The second study used acupuncture points ST34, ST44, and PC5 57 The third used acupuncture points LI4 and HT7, with emergence agitation as secondary endpoint. ${ }^{58}$ One study reported on acupuncture applied to the PC6 acupoint to treat sore throat due to intubation. ${ }^{56}$

Acupuncture or acupressure was initiated before the induction of anesthesia in seven studies, ${ }^{52,53,55,62,64-66}$ during anesthesia in five studies, ${ }^{37,56,58-60}$ and postoperatively in three studies. ${ }^{54,57,61}$ One study tested both preoperative and perioperative acupuncture. ${ }^{63}$

Blinding of patients and assessor occurred in eleven studies out of $16 .^{37,54,55,57,58,60,62-66}$ In four studies, ${ }^{52,53,56,61}$ patient was blinded but blinding of assessors was unclear. One study was open. ${ }^{59}$

For maintenance anesthesia, ten studies used volatile anesthetics, ${ }^{52,54-56,58,60,62-65}$ four studies used intravenous anesthetics, ${ }^{57,59,61,66}$ a multicenter study included both volatile and intravenous anesthetics, ${ }^{37}$ and in one study anesthesia was not specified. ${ }^{53}$ The studies encompassed a range of types of surgery, including strabismus surgery, tonsillectomy, and laparoscopic cholecystectomy.

With regard to POP, one study on pain found no significance between acupuncture and standard care, but a post-hoc analysis found a difference between acupuncture and sham, favoring acupuncture. ${ }^{61}$ Another study found significantly reduced swallowing pain scores for acupuncture and standard care, but not for sham acupuncture. ${ }^{57}$ Lastly, one study found significantly less PONV in the acupressure group compared with in the standard care group, but not compared with the sham group. ${ }^{53}$

There were several secondary outcomes reported. Four studies reported the need for rescue medication ${ }^{52,61,6264,66}$ and five reported adverse events..$^{37,53,57,59,63}$ Discomfort and treatment satisfaction were reported twice. ${ }^{37,59,60,62}$ Resumption to normal activity, complete response, emergence agitation, parent satisfaction, and recovery time were reported once each. ${ }^{53,58,60,62}$ Characteristics of the acupuncture and acupressure studies are presented in Tables 1 and 2.

\section{Discussion}

In our review, we might challenge the definition of "ambulatory anesthesia". Among the included studies, nine studies were presented as being performed in an ambulatory setting. ${ }^{37,53-55,58-60,62,65}$ The remaining seven studies were seemingly performed on inpatients. ${ }^{52,56,57,61,63,64,66}$ However, the type of surgery reported was performed in ambulatory settings elsewhere. For the purpose of our review, these studies were regarded as virtually having been conducted in an ambulatory anesthesia setting. Therefore, we included these studies.

This review include only papers dealing with bodily acupuncture and acupressure, and not other related therapeutic techniques. Other acupuncture-related techniques might be regarded to be in the same category of alternative therapeutic approaches. On the other hand, different acupuncture-related techniques might be very different when compared with bodily acupuncture and acupressure vary greatly. For the purpose of this review, we focused on homogeneity in the selection process.

For this clinical review, and from a practical clinical point of view, acupressure is frequently used for nausea in a wide range of indications, including PONV. Besides, acupressure for nausea is a frequently researched acupuncture-related technique and is combined with bodily acupuncture in scientific studies. Finally, acupressure is an acupuncture-related treatment modality that is easy to implement in a clinical ambulatory setting. Therefore, we included acupressure in our review.

Skin-penetrating body acupuncture might differ from other acupuncture-related techniques in terms of "acupuncture relevance". Some acupuncture-related techniques have quite different therapeutic approaches in terms of point selection, point specificity, skin penetration, theoretical foundation, stimulation intensity, and accuracy. In a clinical setting, skin-penetrating body acupuncture might be regarded as optimal acupuncture stimulation, compared with other acupuncture-related techniques, even though these techniques are considered as acupuncture.

Well aware of this potential for bias, we developed a tool to explore the acupuncture relevance of related techniques. The degree of optimal acupuncture stimulation in accordance with TCM can be expressed by the Optimal Acupuncture Continuum Indicator Summary (OACIS) tool, modified from the Pragmatic-Explanatory Continuum Indicator Summary (PRECIS) tool by Thorpe et al. ${ }^{67}$ Figure 2 provides a concrete picture of the acupuncture relevance of different modalities of acupuncture. Ear acupuncture and electroacupuncture 


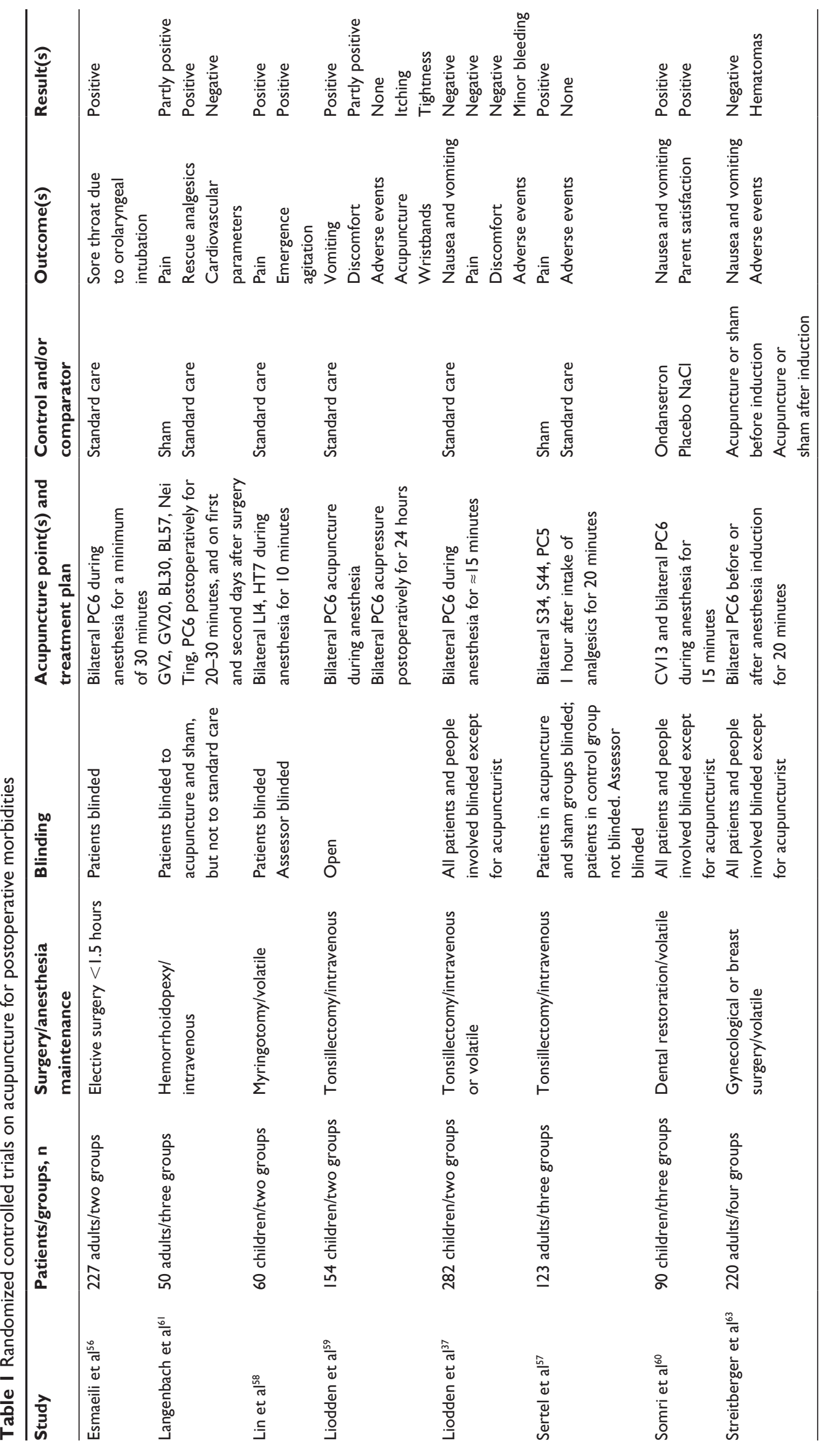




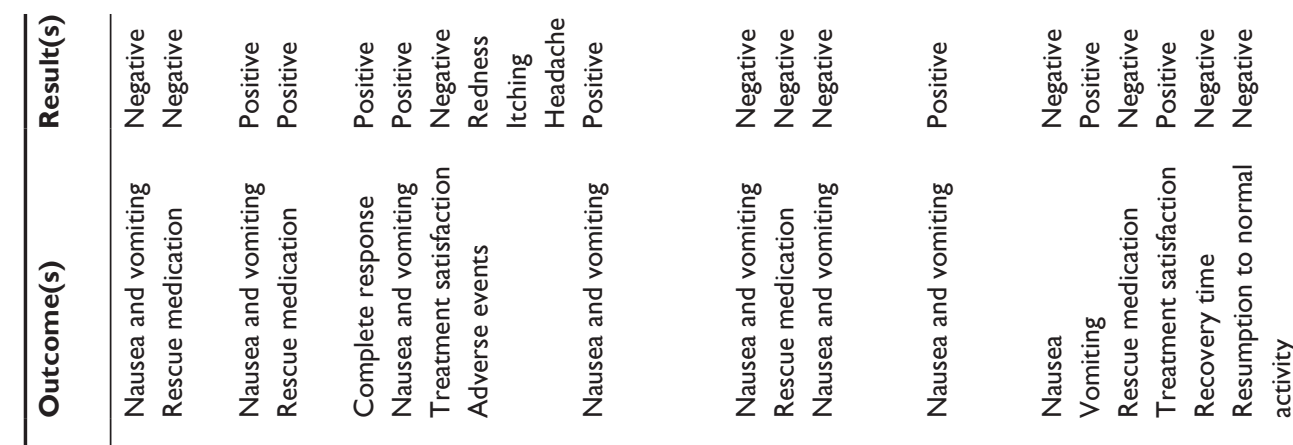

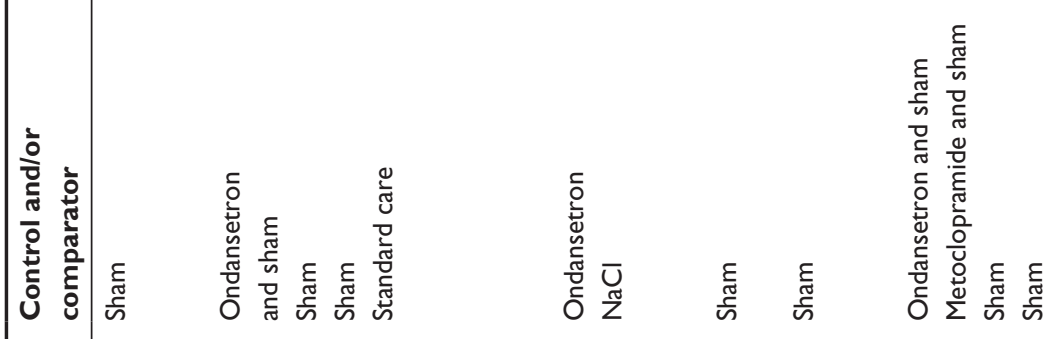

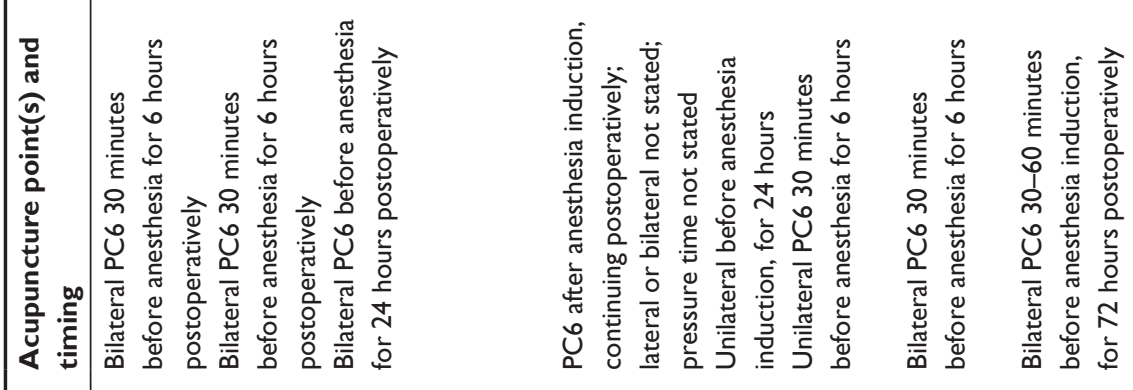

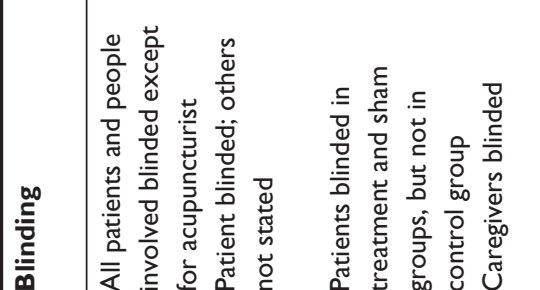
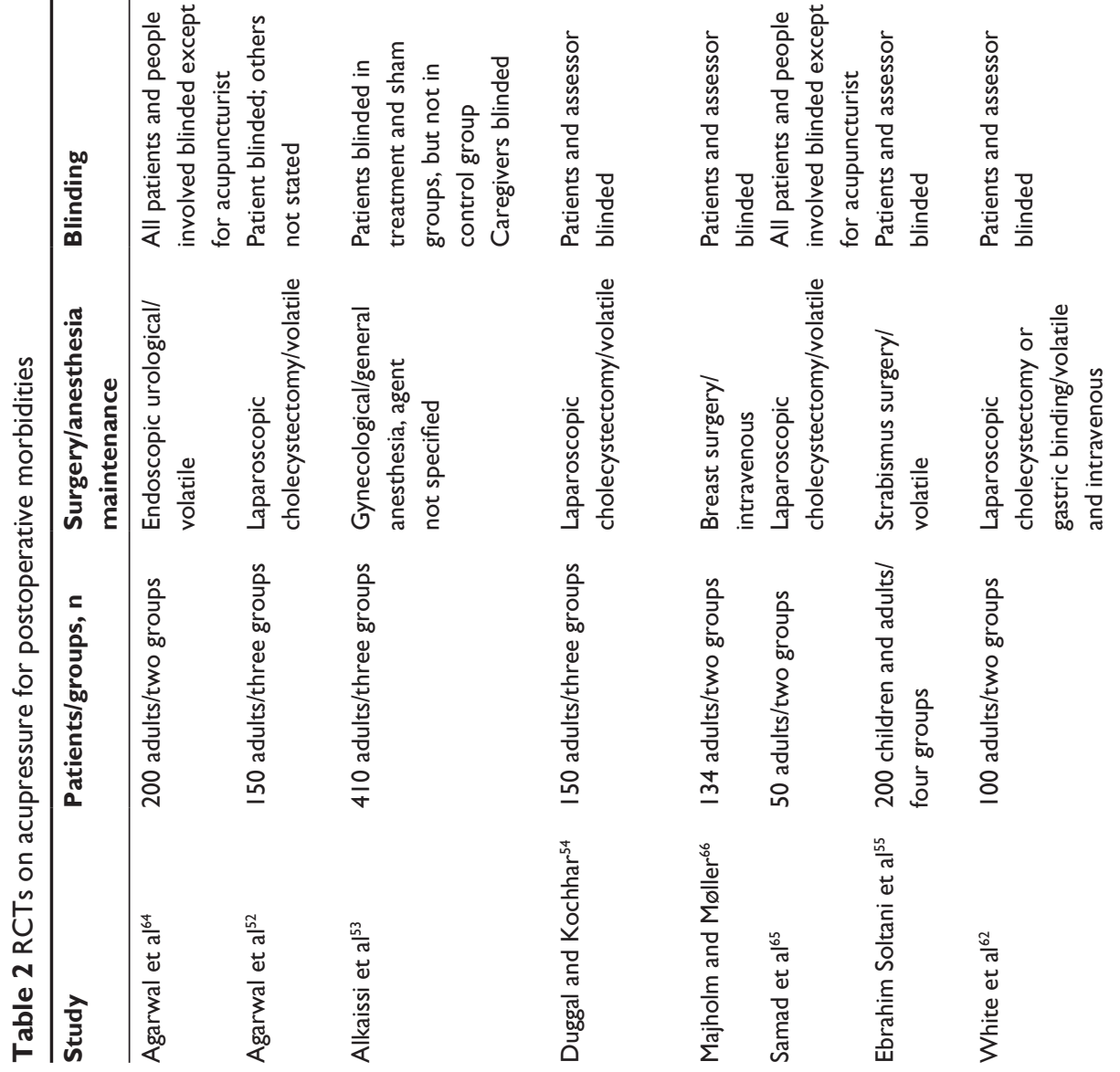

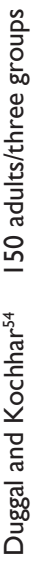

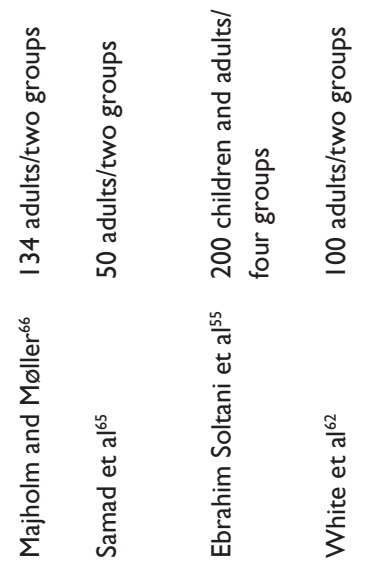


have been selected to represent the variability of stimulation intensities/modalities.

\section{Risk of reporting bias}

In the review process, considering the risk of reporting bias was essential, as bias could have distorted the results and undermined the validity. Further, identified risk factors for nausea and vomiting might also have influenced the results. We admit to having performed a limited search of selected electronic databases. Consequently, we might have missed some eligible RCTs published elsewhere. This may have resulted in selection bias.

Limitation of the search to RCTs and limited publication years may also have resulted in risk of selection bias. Even though we did a thorough search and all authors participated in the selection process, we cannot disregard the possibility that we failed to identify relevant articles.
Furthermore, the subjectivity in the assessing process may have led to risk of selection bias.

Restricting the review according to our selection criteria could have introduced risk of publication bias, as unpublished articles were excluded, and articles not subjected to peer review were likely not identified. Another publication bias, also referred to as the bias against the null hypothesis, is the tendency for published studies to report statistically significant results, as authors tend to refrain from submitting articles with negative results, and peer reviewers tend to reject reviewing, editors tend not to publish, and readers tend to ignore the results of such studies. ${ }^{68}$

We detected one duplicate that might have produced duplicate publication bias. One study was published under different titles in two different journals and years. ${ }^{55,69}$ Lastly, limiting our search to publications in the English language may have risked language bias.

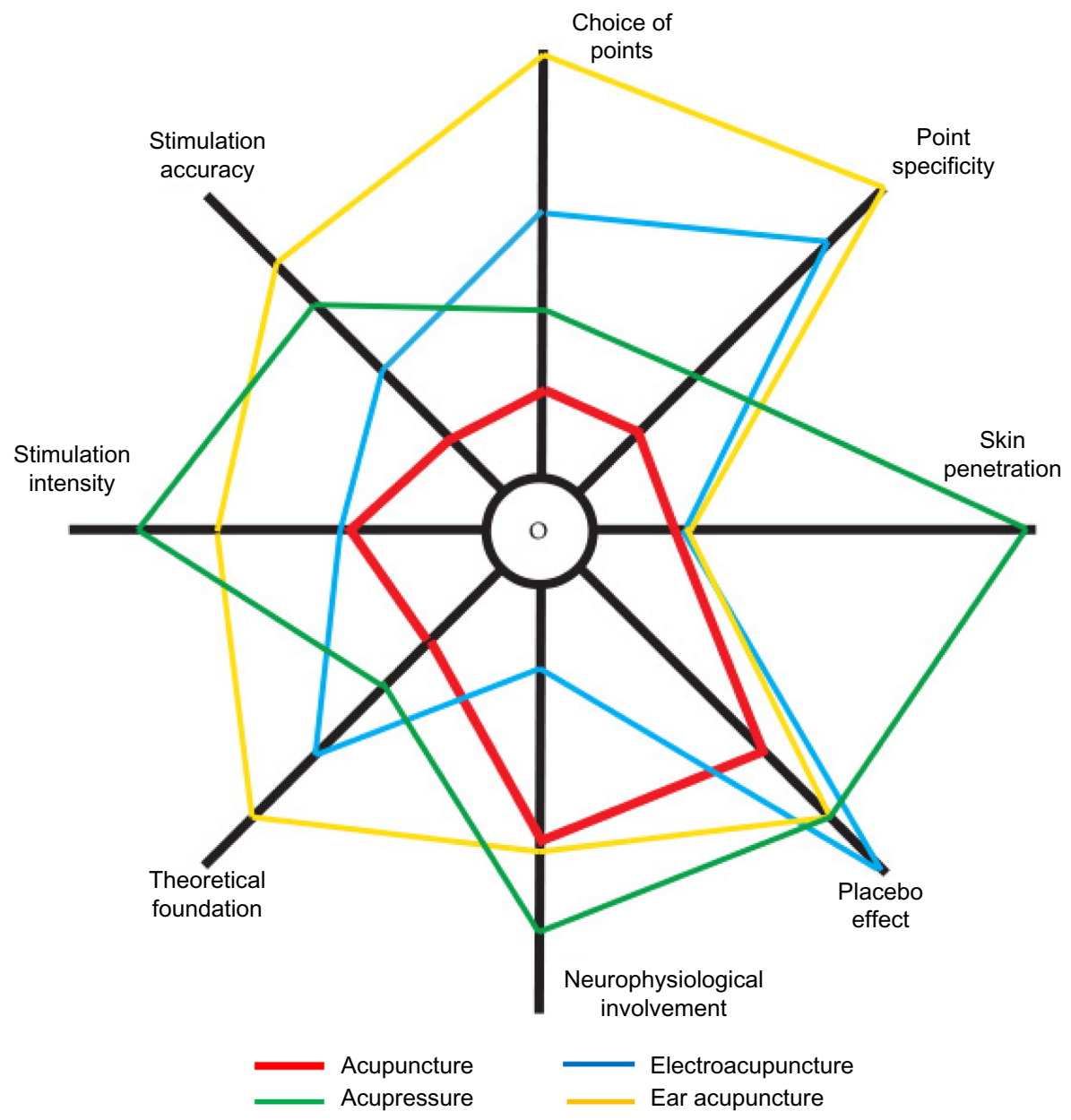

Figure 2 The degree of optimal acupuncture stimulation in accordance with traditional Chinese medicine can be expressed by the Optimal Acupuncture Continuum Indicator Summary (OACIS) wheel. In this figure, the acupuncture relevance of acupuncture-related techniques is compared with skin-penetrating acupuncture needles and acupressure stimulation. The lines from the hub $(\mathrm{O})$ represent the modality continuum of optimal acupuncture-suboptimal acupuncture, illustrated as spokes in a wheel. A suboptimal acupuncture moves outward to the rim, while optimal acupuncture is found nearer to the hub.

Note: Preproduced from: Journal of Clinical Epidemiology, May 2009: Vol:10, Thorpe KE, Zwarenstein M, Oxman AD, et al. A pragmatic-explanatory continuum indicator summary (PRECIS): a tool to help trial designers. 2009; I80(I0):E47-E57. @ 2009, with permission from Elsevier. 


\section{Anesthesia as risk factor for PONV}

Propofol has antiemetic properties, and it is now well established that maintenance of anesthesia with propofol is superior to that with volatile agents, including isoflurane and sevoflurane with or without nitrous oxide, in terms of reducing PONV in adults and children. ${ }^{70-74}$ Among eight studies using volatiles, four found acupuncture effective for PONV, ${ }^{52,54,60}$ and one found acupuncture effective for vomiting, ${ }^{6}$ but not for nausea. One study using intravenous anesthesia found acupuncture effective, ${ }^{59}$ and one found it was not effective. ${ }^{66} \mathrm{It}$ is not possible to draw any conclusions due to the small number of studies reviewed, however, tentatively one may assume that volatiles do not seem to affect the acustimulation interventions.

\section{Surgery as a risk factor for PONV}

Type of surgery as a risk factor for PONV is debated. According to Eberhart et al, ${ }^{75}$ strabismus surgery is considered the only relevant procedure associated with increased risk in infants, while other studies have shown that ear, nose, and throat surgery is highly associated with PONV, especially tonsillectomy. ${ }^{6,76} \mathrm{Habib}$ and $\mathrm{Gan}^{77}$ found in their review that major gynecological, laparoscopic, and breast surgeries were risk factors for PONV. In line with this a recent review by Apfel et a $1^{78}$ also suggest that cholecystectomy, laparoscopic, and gynecological surgeries are significantly associated with the incidence of PONV.

In the present review, among the ten studies on surgery types associated with risk of PONV, ${ }^{37,52-55,57,59,62,63,65}$ six studies found acupuncture effective for PONV. ${ }^{52-55,57,59}$ Another three studies did not find acupuncture effective, ${ }^{37,63,65}$ and one study found acupuncture effective for vomiting, ${ }^{62}$ but not for nausea. In comparison, out of the five studies reporting on surgeries not associated with risk of PONV, four studies found acupuncture effective for PONV, and one study found acupuncture partly effective. It is not possible to draw any conclusions due to the small number of studies reviewed, however, tentatively one may assume that type of surgery does not seem to affect acustimulation interventions.

\section{Quality of acupuncture, acupressure, and point selection in the papers}

Acupuncture treatment and the theory behind it vary across published research studies. This probably reflects the diversity of acupuncture use in clinical contexts worldwide. There are obvious characteristics that are inconsistent, such as location and depth of needle insertion, the rationale for selecting and use of the different acupuncture points, the frequency and number of treatments, and the addition of electrical stimulation or use of laser.

More subtle characteristics are the level of experience and education of the acupuncturist in question. However, after the publication of "Standards for reporting interventions in controlled trials of acupuncture: the STRICTA recommendations" in $2002,{ }^{79}$ followed by the publication of a collaboration between the Consolidated Standards of Reporting Trials (CONSORT) and the Standards for Reporting Interventions in Controlled Trials of Acupuncture (STRICTA) groups ${ }^{80}$ the reporting of acupuncture trials has become better.

Even though the aforementioned issues are raised in several of the reviewed papers, we are left with more questions than answers with regard to the quality of acupuncture, acupressure, and point selection. What is an expert acupuncturist? To what depth should the needles be inserted? For how long should the needles be retained? More detail needs to be gone into about the procedure; it is insufficient to report only that the needles remained in place during surgery and were removed at the end of surgery. It is also common, and indeed relevant for an acupuncturist, to present a rationale for the selection of acupoints.

\section{Other methodology issues}

Eight out of the 16 articles employed a power calculation, ${ }^{37,57-}$ ${ }^{62,66}$ but one study was completed before the sample size was attained, ${ }^{61}$ and in one study the description of the calculation was unclear. ${ }^{57}$ Samples that are too small may be inadequate to provide a statistically significant result.

Only four out of the 16 articles reported adequate allocation concealment. ${ }^{37,59,61,63}$ If allocation concealment is not satisfactory, one can expect a biased estimate of the treatment effect. ${ }^{81}$

\section{Placebo effect in acupuncture/ acupressure studies}

The notion of an ideal placebo control in acupuncture research has yet to be confirmed. Placebo controls commonly used include sham acupuncture (acupuncture on non-acupuncture points), superficial acupuncture (penetrating needling of the skin), and placebo acupuncture needles (needles not penetrating the skin). Common acupressure placebos are wristbands with a felt patch, without a pressure "bead", or application of the bead inappropriately on a non-acupuncture point.

The use of placebo controls is controversial due to their large nonspecific effects, which may imply difficulties in detecting the effects of the intervention and in interpreting 
results. ${ }^{82}$ Another hypothesis is that placebo controls are not inert, but may produce specific effects by evoking physiological responses similar to real acupuncture. ${ }^{83}$ The use of - for example - superficial acupuncture needling as a control intervention, reflects a lack of the historical knowledge of acupuncture theory and usage. This form of needling has been practiced in Japan for a long time and may well have clinical effect. ${ }^{84}$ Consequently, instead of reducing risk of bias, placebo acupuncture may introduce risk of bias against study results.

To remedy this, a third study group may be included: standard care. Any difference between the control and sham groups could be related to placebo responses, including potential nonspecific and specific effects of sham treatment. This was done in three studies in the present review. ${ }^{53,57,61}$ However, the sham procedures in the reviewed papers can definitively be questioned.

Similar wristbands were used in one study ${ }^{53}$ in both intervention groups. The so-called sham was placed on the dorsum of the arm, probably off an acupuncture point. The reports of adverse events in this study showed that this sham procedure was far from a tactile stimulation. Hence, and not unexpectedly, similar stimulation of two different points of the body may have a similar effect on the outcome. This raises an interesting question as to whether or not there are point-specific effects of acupressure; however, this discussion is beyond the scope of this review.

\section{The nature of acupuncture/ acupressure in the studies}

It has been argued that the timing of acustimulation - pre-, per-, or postoperatively - has implications for the effect of the treatment. A review by Wang et $\mathrm{al}^{85}$ indicated that acupuncture is effective when performed pre- and postoperatively. A study by White et $\mathrm{al}^{86}$ showed that acupuncture has little preemptive effect; the optimal timing of stimulation seemed to be postoperatively. It has also been held that acustimulation is ineffective when performed while under anesthesia. ${ }^{85,87}$

Three studies out of four in our review performed acupuncture during anesthesia and argued against this; acupuncture was found effective in preventing sore throat, ${ }^{56}$ in reducing pain and emergence agitation, ${ }^{58}$ and in PONV ${ }^{60}$ The two latter studies involved children. Children are strong reactors and respond well to acupuncture, ${ }^{33}$ and our review found that pediatric acupuncture seemed to be effective in four out of five studies. ${ }^{37,55,58-60}$

The philosophy of TCM is based on non-reductionist thinking, and things are typically seen inseparable as parts from a whole. ${ }^{88}$ Individual diagnoses and individual choice of acupuncture points and stimulation are essential approaches ${ }^{89}$
The complex nature of acupuncture makes research through standard methodological approaches challenging and this sometimes contributes to ambiguous results, ${ }^{90}$ basically finding nothing either way.

Standardized acupuncture and acupressure are easy to perform and do not demand extra time and resources. ${ }^{91}$ The possibility of getting a new treatment implemented in practice depends on, among other things, its feasibility.

Our review found that invasive acupuncture and noninvasive acupressure seem to be equally effective modalities. Given this, acupressure may be a favorable choice, well tolerated by adult patients afraid of needling, and children. Acupressure can be performed by patients, parents, and care providers. Antiemetic drugs and opioids entail several unwanted adverse events, and multimodal approaches to PONV and POP are recommended. ${ }^{10,14,15}$ Acupuncture and acupressure are associated with low cost and a low incidence of adverse events. ${ }^{22}$

\section{The effect of acupuncture/acupressure in postoperative morbidities}

The studies in our review display ambiguous and conflicting results and the evidence is not consistent, as shown in previous reviews and meta-analyses. ${ }^{24,29,87,92,93}$ The majority of the reviewed papers indicate that acupuncture and acupressure may be effective in reducing PONV and POP. This is also in line with the Society for Ambulatory Anesthesia guidelines for the management of PONV. ${ }^{94}$

The reporting of adverse events was sparse; only five studies included adverse events as a secondary outcome. ${ }^{37,53,57,59,63}$ Two acupuncture studies reported hematomas and bleeding, ${ }^{37,63}$ and one study reported itching, tightness, redness, and headache from acupressure. ${ }^{53}$ This is in line with previous research. ${ }^{35}$

However, it is challenging to aggregate results from single studies into a common understanding of an overall effect in acupuncture and/or acupressure for morbidities in the ambulatory setting. Systematic reviews and metaanalyses might well achieve a higher statistical power, although by including methodologically weak studies, but will still result in bad statistics. Our review process has also revealed that a lack of knowledge and clinical experience of acupuncture might bias a review or meta-perspective of the topic.

\section{Conclusion}

This paper presents an overview of the currently available relevant literature on acupuncture/acupressure in an ambula- 
tory setting. Numerous papers describe the use of acupuncture and acupuncture-related techniques for postoperative morbidities in ambulatory anesthesia; however, only a few are high-quality RCTs in acupuncture/acupressure.

A major proportion of the reviewed papers highlight an effect for postoperative morbidities in an ambulatory setting. Some studies also indicate that the effect of acupuncture and acupressure was similar to that of antiemetic drugs. It is therefore reasonable to consider acupuncture and/or acupressure as part of ambulatory anesthesia. This is also supported by the fact that treatment costs are low and adverse effects are rare.

However, one should bear in mind that research on acupuncture/acupressure in an ambulatory setting contributes to ambiguous conclusions. There is substantial research to support the effect of acupuncture/acupressure for PONV. On the other hand, acupuncture/acupressure for other postoperative morbidities in an ambulatory setting can still not be regarded as evidence-based practice and more reliable and valid research is warranted.

\section{Recommendations for future research}

Research on complex therapies, such as acupuncture, should be conducted with an approach toward the whole treatment package, including placebo responses, using a pragmatic design. Research methodology should be rigorous and have a specific focus toward blinding, placebo, and allocation concealment. Conventional precautions such as unbiased selection, power calculation, homogeneity, and clearly defined outcomes must be fulfilled to ensure validity and reliability. Research involving acupuncture should also adhere to methodological and reporting principles described in the CONSORT and STRICTA guidelines. ${ }^{79,80}$

\section{Disclosure}

The authors declare no conflicts of interest in this work.

\section{References}

1. Parra-Sanchez I, Abdallah R, You J, et al. A time-motion economic analysis of postoperative nausea and vomiting in ambulatory surgery. Can J Anaesth. 2012;59(4):366-375.

2. White PF, Kehlet H. Improving postoperative pain management: what are the unresolved issues? Anesthesiology. 2010;112(1):220-225.

3. Comité douleur-anesthésie locorégionale et le comité des référentiels de la Sfar. [Formalized recommendations of experts 2008. Management of postoperative pain in adults and children.] Ann Fr Anesth Reanim. 2008;27(12):1035-1041. French.

4. Apfel CC, Korttila K, Abdalla M, et al; IMPACT Investigators. A factorial trial of six interventions for the prevention of postoperative nausea and vomiting. $N$ Engl J Med. 2004;350(24):2441-2451.
5. Stadler M, Bardiau F, Seidel L, Albert A, Boogaerts JG. Difference in risk factors for postoperative nausea and vomiting. Anesthesiology. 2003;98(1):46-52.

6. Elgueta MF, Echevarría GC, De la Fuente N, et al. Effect of intravenous fluid therapy on postoperative vomiting in children undergoing tonsillectomy. Br J Anaesth. 2013;110(4):607-614.

7. McGrath B, Elgendy H, Chung F, Kamming D, Curti B, King S. Thirty percent of patients have moderate to severe pain $24 \mathrm{hr}$ after ambulatory surgery: a survey of 5,703 patients. Can J Anaesth. 2004;51(9):886-891.

8. Tufano R, Puntillo F, Draisci G, et al. ITalian Observational Study of the management of mild-to-moderate Post-Operative Pain (ITOSPOP). Minerva Anestesiol. 2012;78(1):15-25.

9. Scuderi PE. Pharmacology of antiemetics. Int Anesthesiol Clin. 2003; 41(4):41-66.

10. Gan TJ, Diemunsch P, Habib AS, et al; Society for Ambulatory Anesthesia. Consensus guidelines for the management of postoperative nausea and vomiting. Anesth Analg. 2014;118(1):85-113.

11. Roberts GW, BekkerTB, Carlsen HH, MoffattCH, Slattery PJ, McClureAF. Postoperative nausea and vomiting are strongly influenced by postoperative opioid use in a dose-related manner. Anesth Analg. 2005; 101(5):1343-1348.

12. Lanza FL, Chan FK, Quigley EM; Practice Parameters Committee of the American College of Gastroenterology. Guidelines for prevention of NSAID-related ulcer complications. Am J Gastroenterol. 2009; 104(3):728-738.

13. Kehlet H, Dahl JB. The value of "multimodal" or "balanced analgesia" in postoperative pain treatment. Anesth Analg. 1993;77(5):1048-1056.

14. Chandrakantan A, Glass PS. Multimodal therapies for postoperative nausea and vomiting, and pain. Br J Anaesth. 2011;107 Suppl 1:i27-i40.

15. Yaster M. Multimodal analgesia in children. Eur J Anaesthesiol. 2010;27(10):851-857.

16. Frass M, Strassl RP, Friehs H, Müllner M, Kundi M, Kaye AD. Use and acceptance of complementary and alternative medicine among the general population and medical personnel: a systematic review. Ochsner J. 2012;12(1):45-56.

17. Zuzak TJ, Boňková J, Careddu D, et al. Use of complementary and alternative medicine by children in Europe: published data and expert perspectives. Complement Ther Med. 2013;21 Suppl 1:S34-S47.

18. Faircloth A. Perceptions of Acupuncture and Acupressure by Anesthesia Providers [dissertation]. Virginia Commonwealth University (VCU) Theses and Dissertations Paper 3586. Richmond, VA: VCU; 2014. Available from: http://scholarscompass.vcu.edu/etd/3586/. Accessed June 26, 2015.

19. Matthews A, Haas DM, O’Mathúna DP, Dowswell T, Doyle M. Interventions for nausea and vomiting in early pregnancy. Cochrane Database Syst Rev. 2014;3:CD007575.

20. Garcia MK, McQuade J, Haddad R, et al. Systematic review of acupuncture in cancer care: a synthesis of the evidence. J Clin Oncol. 2013; 31(7):952-960.

21. Lee A, Fan LT. Stimulation of the wrist acupuncture point P6 for preventing postoperative nausea and vomiting. Cochrane Database Syst Rev. 2009(2):1-86.

22. Cheong KB, Zhang JP, Huang Y, Zhang ZJ. The effectiveness of acupuncture in prevention and treatment of postoperative nausea and vomiting - a systematic review and meta-analysis. PLoS One. 2013; 8(12):e82474.

23. Dune LS, Shiao SY. Metaanalysis of acustimulation effects on postoperative nausea and vomiting in children. Explore (NY). 2006;2(4): 314-320.

24. Lee MS, Ernst E. Acupuncture for surgical conditions: an overview of systematic reviews. Int J Clin Pract. 2014;68(6):783-789.

25. Ezzo J, Streitberger K, Schneider A. Cochrane systematic reviews examine P6 acupuncture-point stimulation for nausea and vomiting. J Altern Complement Med. 2006;12(5):489-495.

26. Nunley C, Wakim J, Guinn C. The effects of stimulation of acupressure point $\mathrm{p} 6$ on postoperative nausea and vomiting: a review of literature. J Perianesth Nurs. 2008;23(4):247-261. 
27. Bolton CM, Myles PS, Nolan T, Sterne JA. Prophylaxis of postoperative vomiting in children undergoing tonsillectomy: a systematic review and meta-analysis. Br J Anaesth. 2006;97(5):593-604.

28. Cho YH, Kim CK, Heo KH, et al. Acupuncture for acute postoperative pain after back surgery: a systematic review and meta-analysis of randomized controlled trials. Pain Pract. 2015;15(3):279-291.

29. Sun Y, Gan TJ, Dubose JW, Habib AS. Acupuncture and related techniques for postoperative pain: a systematic review of randomized controlled trials. Br J Anaesth. 2008;101(2):151-160.

30. Liodden I, Norheim AJ. Acupuncture and related techniques in ambulatory anesthesia. Curr Opin Anaesthesiol. 2013;26(6):661-668.

31. O'Connell NE, Wand BM, Goldacre B. Interpretive bias in acupuncture research?: A case study. Eval Health Prof. 2009;32(4):393-409.

32. Pandolfi M. The autumn of acupuncture. Eur J Intern Med. 2012; 23(1):31-33.

33. Streitberger K, Kranke P. Evidence for the efficacy of acupressure for preventing post-operative nausea and vomiting: an ongoing debate. Eur J Anaesthesiol. 2011;28(6):396-398.

34. Wu C, Weber W, Kozak L, et al. A survey of complementary and alternative medicine (CAM) awareness among neurosurgeons in Washington State. J Altern Complement Med. 2009;15(5):551-555.

35. Ernst E, White AR. Prospective studies of the safety of acupuncture: a systematic review. Am J Med. 2001;110(6):481-485.

36. Jindal V, Ge A, Mansky PJ. Safety and efficacy of acupuncture in children: a review of the evidence. J Pediatr Hematol Oncol. 2008; 30(6):431-442.

37. Liodden I, Sandvik L, Valeberg BT, Borud E, Norheim AJ. Acupuncture versus usual care for postoperative nausea and vomiting in children after tonsillectomy/adenoidectomy: a pragmatic, multicentre, double-blinded, randomised trial. Acupunct Med. 2015;33(3):196-203.

38. ChenZY,LinL, Wang HH, etal. Ondansetron combined with ST36(Zusanli) acupuncture point injection for postoperative vomiting. Acupunct Med. 2014;32(2):124-131

39. Wang SM, Kain ZN. P6 acupoint injections are as effective as droperidol in controlling early postoperative nausea and vomiting in children. Anesthesiology. 2002;97(2):359-366.

40. Rusy LM, Hoffman GM, Weisman SJ. Electroacupuncture prophylaxis of postoperative nausea and vomiting following pediatric tonsillectomy with or without adenoidectomy. Anesthesiology. 2002;96(2): 300-305.

41. Yeh BY, Hsu YC, Huang JY, et al. Effect of electroacupuncture in postanesthetic shivering during regional anesthesia: a randomized controlled trial. BMC complement Altern Med. 2012;12:233.

42. Wang H, Xie Y, Zhang Q, et al. Transcutaneous electric acupoint stimulation reduces intra-operative remifentanil consumption and alleviates postoperative side-effects in patients undergoing sinusotomy: a prospective, randomized, placebo-controlled trial. $\mathrm{Br} J$ Anaesth. 2014;112(6):1075-1082.

43. Zhang Q, Gao Z, Wang H, et al. The effect of pre-treatment with transcutaneous electrical acupoint stimulation on the quality of recovery after ambulatory breast surgery: a prospective, randomised controlled trial. Anaesthesia. 2014;69(8):832-839.

44. Holzer A, Leitgeb U, Spacek A, Wenzl R, Herkner H, Kettner S. Auricular acupuncture for postoperative pain after gynecological surgery: a randomized controlled trail. Minerva Anestesiol. 2011;77(3):298-304.

45. Usichenko TI, Kuchling S, Witstruck T, et al. Auricular acupuncture for pain relief after ambulatory knee surgery: a randomized trial. CMAJ. 2007;176(2):179-183.

46. Schlager A, Boehler M, Pühringer F. Korean hand acupressure reduces postoperative vomiting in children after strabismus surgery. $\mathrm{Br} J$ Anaesth. 2000;85(2):267-270.

47. Boehler M, Mitterschiffthaler G, Schlager A. Korean hand acupressure reduces postoperative nausea and vomiting after gynecological laparoscopic surgery. Anesth Analg. 2002;94(4):872-875, table of contents.

48. Kim KS, Kim DW, Yu YK. The effect of capsicum plaster in pain after inguinal hernia repair in children. Paediatr Anaesth. 2006;16(10): 1036-1041.
49. Acar HV, Yilmaz A, Demir G, Günal Eruyar S, Dikmen B. Capsicum plasters on acupoints decrease the incidence of emergence agitation in pediatric patients. Paediatr Anaesth . 2012;22(11):1105-1109.

50. Kaptchuk T. The Web that has No Weaver: Understanding Chinese Medicine. London: Rider; 1983.

51. A standard international acupuncture nomenclature: memorandum from a WHO meeting. Bull World Health Organ. 1990;68(2):165-169.

52. Agarwal A, Bose N, Gaur A, Singh U, Gupta MK, Singh D. Acupressure and ondansetron for postoperative nausea and vomiting after laparoscopic cholecystectomy. Can J Anaesth. 2002;49(6):554-560.

53. Alkaissi A, Evertsson K, Johnsson VA, Ofenbartl L, Kalman S. P6 acupressure may relieve nausea and vomiting after gynecological surgery: an effectiveness study in 410 women. Can J Anaesth. 2002;49(10): 1034-1039.

54. Duggal G, Kochhar S. Comparative study of ondansetron and acupressure for postoperative nausea and vomiting after laparoscopic cholecystectomy. Journal of Advance Researches in Biological Sciences. 2012;4(4):330-336.

55. Ebrahim Soltani A, Mohammadinasab H, Goudarzi M, et al. Acupressure using ondansetron versus metoclopramide on reduction of postoperative nausea and vomiting after strabismus surgery. Arch Iran Med. 2010;13(4):288-293.

56. Esmaeili S, Alizadeh R, Shoar S, Naderan M, Shoar N. Acupuncture in preventing postoperative anaesthesia-related sore throat: a comparison with no acupuncture. Acupunct Med. 2013;31(3):272-275.

57. Sertel S, Herrmann S, Greten HJ, et al. Additional use of acupuncture to NSAID effectively reduces post-tonsillectomy pain. Eur Arch Otorhinolaryngol. 2009;266(6):919-925.

58. Lin YC, Tassone RF, Jahng S, et al. Acupuncture management of pain and emergence agitation in children after bilateral myringotomy and tympanostomy tube insertion. Paediatr Anaesth. 2009;19(11):1096-1101.

59. Liodden I, Howley M, Grimsgaard AS, et al. Perioperative acupuncture and postoperative acupressure can prevent postoperative vomiting following paediatric tonsillectomy or adenoidectomy: a pragmatic randomised controlled trial. Acupunct Med. 2011;29(1):9-15.

60. Somri M, Vaida SJ, Sabo E, Yassain G, Gankin I, Gaitini LA. Acupuncture versus ondansetron in the prevention of postoperative vomiting. A study of children undergoing dental surgery. Anaesthesia. 2001; 56(10):927-932.

61. Langenbach MR, Aydemir-Dogruyol K, Issel R, Sauerland S. Randomized sham-controlled trial of acupuncture for postoperative pain control after stapled haemorrhoidopexy. Colorectal Dis. 2012;14(8):e486-e491.

62. White PF, Zhao M, Tang J, et al. Use of a disposable acupressure device as part of a multimodal antiemetic strategy for reducing postoperative nausea and vomiting. Anesth Analg. 2012;115(1):31-37.

63. Streitberger K, Diefenbacher M, Bauer A, et al. Acupuncture compared to placebo-acupuncture for postoperative nausea and vomiting prophylaxis: a randomised placebo-controlled patient and observer blind trial. Anaesthesia. 2004;59(2):142-149.

64. Agarwal A, Pathak A, Gaur A. Acupressure wristbands do not prevent postoperative nausea and vomiting after urological endoscopic surgery. Can J Anaesth. 2000;47(4):319-324.

65. Samad K, Afshan G, Kamal R. Effect of acupressure on postoperative nausea and vomiting in laparoscopic cholecystectomy. J Pak Med Assoc. 2003;53(2):68-72.

66. Majholm B, Møller AM. Acupressure at acupoint P6 for prevention of postoperative nausea and vomiting: a randomised clinical trial. Eur J Anaesthesiol. 2011;28(6):412-419.

67. Thorpe KE, Zwarenstein M, Oxman AD, et al. A pragmatic-explanatory continuum indicator summary (PRECIS): a tool to help trial designers. CMAJ. 2009;180(10):E47-E57.

68. Conn VS, Valentine JC, Cooper HM, Rantz MJ. Grey literature in meta-analyses. Nurs Res. 2003;52(4):256-261.

69. Ebrahim Soltani AR, Mohammadinasab H, Goudarzi M, et al. Comparing the efficacy of prophylactic p6 acupressure, ondansetron, metoclopramide and placebo in the prevention of vomiting and nausea after strabismus surgery. Acta Med Iran. 2011;49(4):208-212. 
70. Deng X, Zhu T. Clinical comparison of propofol-remifentanil TCI with sevoflurane induction/maintenance anesthesia in laparoscopic cholecystectomy. Pak J Med Sci. 2014;30(5):1017-1021.

71. Chui J, Mariappan R, Mehta J, Manninen P, Venkatraghavan L. Comparison of propofol and volatile agents for maintenance of anesthesia during elective craniotomy procedures: systematic review and meta-analysis. Can J Anaesth. 2014;61(4):347-356.

72. Chung JH, Kim YH, Ko YK, Lee SY, Nam YT, Yoon SH. Vomiting after a pediatric adenotonsillectomy: comparison between propofol induced sevoflurane-nitrous oxide maintained anesthesia and TIVA with propofol-remifentanil. Korean J Anesthesiol. 2010;59(3):185-189.

73. Sneyd JR, Carr A, Byrom WD, Bilski AJ. A meta-analysis of nausea and vomiting following maintenance of anaesthesia with propofol or inhalational agents. Eur J Anaesthesiol. 1998;15(4):433-445.

74. Hofer CK, Zollinger A, Büchi S, et al. Patient well-being after general anaesthesia: a prospective, randomized, controlled multi-centre trial comparing intravenous and inhalation anaesthesia. Br J Anaesth. 2003; 91(5):631-637.

75. Eberhart LH, Geldner G, Kranke P, et al. The development and validation of a risk score to predict the probability of postoperative vomiting in pediatric patients. Anesth Analg. 2004;99(6):1630-1637.

76. Raeder J. Postoperativ kvalme og oppkast [Postoperative nausea and vomiting]. Tidsskr Nor Laegeforen. 2005;125(13):1831-1832. Norwegian.

77. Habib AS, Gan TJ. Evidence-based management of postoperative nausea and vomiting: a review. Can J Anaesth. 2004;51(4):326-341.

78. Apfel CC, Heidrich FM, Jukar-Rao S, et al. Evidence-based analysis of risk factors for postoperative nausea and vomiting. Br J Anaesth. 2012;109(5):742-753.

79. MacPherson H, White A, Cummings M, Jobst KA, Rose K, Niemtzow RC. Standards for reporting interventions in controlled trials of acupuncture: the STRICTA recommendations. J Altern Complement Med. 2002;8(1):85-89.

80. Prady SL, Richmond SJ, Morton VM, Macpherson H. A systematic evaluation of the impact of STRICTA and CONSORT recommendations on quality of reporting for acupuncture trials. PLoS One. 2008;3(2):e1577.

81. Polit DF, Beck CT. Nursing Research: Generating and Assessing Evidence for Nursing Practice 8th Ed. Philadelphia, PA: Lippincott Williams \& Wilkins; 2008.
82. Linde K, Niemann K, Schneider A, Meissner K. How large are the nonspecific effects of acupuncture? A meta-analysis of randomized controlled trials. BMC Med. 2010;8:75.

83. Lundeberg T, Lund I, Näslund J, Thomas M. The Emperor's sham wrong assumption that sham needling is sham. Acupunct Med. 2008;26(4):239-242.

84. Alraek T, Birch S. Commentary: acupuncture research strategies. Forsch Komplementmed. 2012;19:43-48.

85. Wang SM, Kain ZN, White PF. Acupuncture analgesia: II. Clinical considerations. Anesth Analg. 2008;106(2):611-621.

86. White PF, Hamza MA, Recart A, et al. Optimal timing of acustimulation for antiemetic prophylaxis as an adjunct to ondansetron in patients undergoing plastic surgery. Anesth Analg. 2005;100(2):367-372.

87. Chernyak GV, Sessler DI. Perioperative acupuncture and related techniques. Anesthesiology. 2005;102(5):1031-1049.

88. Birch S, Alraek T. Traditional East Asian medicine: how to understand and approach diagnostic findings and patterns in a modern scientific framework? Chin J Integr Med. 2014;20(5):336-340.

89. Ritenbaugh C, Verhoef M, Fleishman S, Boon H, Leis A. Whole systems research: a discipline for studying complementary and alternative medicine. Altern Ther Health Med. 2003;9(4):32-36.

90. Fønnebø V, Grimsgaard S, Walach H, et al. Researching complementary and alternative treatments - the gatekeepers are not at home. BMC Med Res Methodol. 2007;7:7.

91. Norheim AJ, Liodden I, Howley M. Implementation of acupuncture and acupressure under surgical procedures in children: a pilot study. Acupunct Med. 2010;28(2):71-73.

92. Madsen MV, Gøtzsche PC, Hróbjartsson A. Acupuncture treatment for pain: systematic review of randomised clinical trials with acupuncture, placebo acupuncture, and no acupuncture groups. BMJ. 2009; 338:a3115.

93. Libonate J, Evans S, Tsao JC. Efficacy of acupuncture for health conditions in children: a review. ScientificWorldJournal. 2008;8:670-682.

94. Gan TJ, Meyer TA, Apfel CC, et al; Society for Ambulatory Anesthesia. Society for Ambulatory Anesthesia guidelines for the management of postoperative nausea and vomiting. Anesth Analg. 2007;105(6):1615-1628.
Ambulatory Anesthesia

\section{Publish your work in this journal}

Ambulatory Anesthesia is an international, peer reviewed, open access journal publishing articles that address all aspects of ambulatory anesthesia practice, in particular: anesthetic techniques, sedation and safety practices, pharmacokinetics, preoperative evaluation, analgesia interventions, regulatory and compliance issues, postoperative recovery,

\section{Dovepress}

patient satisfaction, administrative topics, and cost analysis themes. The manuscript management system is completely online and includes a very quick and fair peer review system, which is all easy to use. Visit http://www.dovepress.com/testimonials.php to read real quotes from published authors. 